\title{
Uma Representação Visual para Gerenciamento de Emergências em Ambientes Fechados
}

\section{Alternative Title: A Visual Representation for Indoor Emergency Management}

\author{
Flavio Nunes \\ Instituto Federal da Bahia \\ Rua Emídio dos Santos, s/n \\ Salvador, Brasil \\ flavionunes@ifba.edu.br
}

\author{
Fernando Teles \\ Instituto Federal da Bahia \\ Rua Emídio dos Santos, s/n \\ Salvador, Brasil \\ fernandoteles@ifba.edu.br
}

\author{
Renato Novais \\ Instituto Federal da Bahia \\ Rua Emídio dos Santos, s/n \\ Salvador, Brasil \\ renato@ifba.edu.br
}

\author{
Sandro Andrade \\ Instituto Federal da Bahia \\ Rua Emídio dos Santos, s/n \\ Salvador, Brasil \\ Manoel Neto \\ Instituto Federal da Bahia \\ Rua Emídio dos Santos, s/n \\ Salvador, Brasil \\ sandroandrade@ifba.edu.bmanoelnetom@ifba.edu.br
}

\begin{abstract}
RESUMO
O ser humano passa muito tempo em ambientes fechados (indoor), tais como escolas, fábricas e empresas. O gerenciamento de emergências em tais ambientes é útil e desafiador. As tecnologias de localização e visualização nestes ambientes não estão tão avançadas quanto às existentes para ambientes abertos (ex: localização via GPS, visualização em mapas, etc). Atualmente, muito esforço é dedicado à localização de pessoas em ambientes indoor. Apesar da relevância dessa atividade, poucos estudos investigam aspectos referentes à visualização desta informação. Este trabalho apresenta uma visualização para ambientes indoor visando o suporte à emergência. A visualização proposta foi baseada na opinião de profissionais da área de emergência, desenvolvida em um protótipo e validada com usuários finais. Os resultados mostram que a visualização desenvolvida é efetiva na transmissão da informação a respeito de um possível cenário de emergência em ambientes fechados, podendo assim dar suporte aos profissionais da área em suas atividades de resgate.
\end{abstract}

\section{Palavras-Chave}

Representação visual, Ambiente fechado, Emergência

\begin{abstract}
Human beings spend much time indoors, such as schools, factories and businesses. The emergency management in such environments is useful and challenging. The location and visualization technologies in these environments are not as advanced as existing for open environments (e.g., location
\end{abstract}

Permission to make digital or hard copies of all or part of this work for personal or classroom use is granted without fee provided that copies are not made or distributed for profit or commercial advantage and that copies bear this notice and the full citation on the first page. To copy otherwise, to republish, to post on servers or to redistribute to lists, requires prior specific permission and/or a fee.

SBSI 2016, May 17th-20th, 2016, Florianópolis, Santa Catarina, Brazil Copyright SBC 2016. via GPS, visualization in maps). Currently, much effort is devoted to identify people in indoor environments. Despite the relevance of this activity, few studies investigate aspects related to the visualization of this information. This paper presents a new visualization for indoor environments aimed at supporting the emergency. The proposed visualization was based on the opinion of emergency professionals, developed as a prototype and validated with end users. The results show that the developed visualization is effective in transmitting information regarding a possible indoors-emergency scenario and can thus support the professionals in their rescue activities.

\section{Categories and Subject Descriptors}

H.4 [Information Systems Applications]: Miscellaneous

\section{General Terms}

Theory, Visualization

\section{Keywords}

Visual Representation, indoor environment, emergency

\section{INTRODUÇÃO}

O ser humano vive a maior parte da sua vida em ambientes fechados, tais como escolas, fábricas e empresas. Estudos reportam que pessoas chegam a passar de $80 \%$ [17] a $92 \%$ [6] do seu tempo em locais fechados. Esta realidade traz à tona preocupações referentes à segurança nestes ambientes, uma vez que acidentes podem impactar vidas humanas. Desta forma, é de grande valia a existência de soluções computacionais que auxiliem forças operacionais na atuação em uma emergência em ambientes fechados.

Emergência é uma "situação que exige uma intervenção imediata de profissionais treinados com equipamentos adequados, para que danos e prejuízos sejam evitados ou minimizados" [15]. Dentre os desafios presentes em uma situação de emergência, destaca-se a identificação da localização das pessoas e a compreensão da situação emergencial. A compreensão pode ser alcançada através de visualização de um 
mapa situacional da emergência. As tecnologias de localização e visualização em ambientes abertos (outdoor) já estão de certa forma consolidadas. Muitos trabalhos utilizam localização via GPS e visualização em mapas [7]. Por outro lado, o mesmo não pode ser dito em relação aos ambientes fechados (indoor). Tanto a localização quanto a visualização ainda têm problemas em aberto.

Atualmente, muito esforço de pesquisa é dedicado à localização de pessoas em ambientes [18][11][4]. Esta é de fato uma tarefa desafiadora para sistemas com este objetivo. Entretanto, a compreensão (i.e., visualização) da situação emergencial é também importante, uma vez que decisões precisam ser tomadas de forma rápida e correta. Infelizmente, não encontra-se na literatura avaliações da efetividade das visualizações desenvolvidas para ambientes fechados, assim como existem em ambientes abertos (e.g., [12]).

Alguns trabalhos trazem exemplos de visualização para ambientes indoor [14][20]. Entretanto, não houve nos trabalhos encontrados uma avaliação experimental da efetividade das soluções visuais propostas.

Este trabalho apresenta uma solução visual para suporte à tomada de decisão em situações emergenciais em ambientes fechados. Foi desenvolvido um protótipo web, chamado VisEM (Visualização de EMergência), que representa a planta baixa de um ambiente, destaca as pessoas e suas características, além de mostrar o histórico espacial das pessoas no ambiente sendo monitorado. Com a VisEM, os profissionais da área de salvamento e gerenciamento de emergências podem tomar ações com um maior nível de precisão por meio da análise visual.

Este trabalho está inserido no contexto de um projeto de um sistema maior, chamado Rescuer Indoor (RI). O RI tem como objetivo construir uma infraestrutura escalável para identificação e visualização de pessoas em ambientes indoor, tendo como foco o suporte ao gerenciamento de emergências. O módulo de visualização foi projetado e desenvolvido tendo como base a opinião de profissionais da área. Profissionais, tais como bombeiros, destacaram questões de prioridade em um salvamento, as quais formaram o foco da solução VisEM.

Uma avaliação foi conduzida para analisar a utilidade das representações visuais criadas pela VisEM. Através do estudo foi possível observar a efetividade da ferramenta em um contexto de salvamento. Porém, os participantes do estudo sugeriram alterações para tornar as representações visuais mais compreensíveis em um contexto emergencial. Os resultados do estudo contribuíram para a evolução da VisEM.

O restante deste trabalho está organizado como segue. A Seção 2 descreve a solução visual para gerenciamento de emergências em ambientes fechados. A Seção 3 destaca o estudo realizado para avaliação da VisEM. A Seção 4 apresenta os novos critérios para representação visual de pessoas. A Seção 5 apresenta alguns trabalhos relacionados. Por fim, a Seção 6 conclui este trabalho destacando possibilidades de trabalhos futuros.

\section{VISEM: UMA SOLUÇÃO VISUAL PARA GERENCIAMENTO DE EMERGÊNCIAS EM AMBIENTES INDOOR}

A VisEM é uma solução visual para gerenciamento de emergências em ambientes indoor ${ }^{1}$. A VisEM faz parte do

\footnotetext{
${ }^{1}$ Mais detalhes da VisEM e do estudo conduzido podem ser
}

Sistema de Gerenciamento de Emergência para Ambientes Fechados (Rescuer Indoor - RI), que coleta, armazena e processa dados referentes a um ambiente fechado. Os sensores $^{2}$ colhem dados de um ambiente fechado monitorado. Esses dados são armazenados em uma base de dados e posteriormente consumidos pela VisEM para representação das informações de sensores e da planta baixa e montagem da visualização do ambiente.

A VisEM foi construída tendo os seguintes requisitos: O1) ser eficaz do ponto de vista da compreensão da situação emergencial em um ambiente fechado. Os usuários da VisEM devem ser capaz de utilizá-la e extrair a informação correta do contexto de emergência; O2) transmitir a informação para os usuários de forma eficiente. Considera-se que a VisEM será eficiente se seus usuários forem capaz de compreender o ambiente em um tempo aceitável para o contexto de emergência; O3) atender a critérios de priorização de resgates, coletados com especialistas da área; e O4) ser flexível e configurável para que seja passível de uso em outros ambientes de gerenciamento de emergências. Para atender aos itens O1 e O2, princípios da área de visualização de informação [16] foram adotados no projeto da VisEM. O estudo apresentado na Seção 3 teve como objetivo avaliar essa efetividade em termos de eficácia e eficiência. A estratégia utilizada para atingir os objetivos O3 e $\mathrm{O} 4$ são explicados em mais detalhes, respectivamente, nas duas subseções seguintes.

\subsection{Priorização de resgate}

Foram conduzidas entrevistas com especialistas em resgate no intuito de entender como se dá o atendimento a possíveis vítimas em uma emergência. De uma forma geral, as forças operacionais buscam dar prioridade às pessoas mais vulneráveis. Neste trabalho, foram considerados três atributos reais acerca das pessoas: idade, sendo que criança tem prioridade sobre idoso, e este sobre adulto; pessoas paradas ou em movimento, sendo que pessoas paradas há mais tempo em um mesmo local podem indicar uma necessidade de socorro prioritário; e portador ou não de necessidades especiais, onde os portadores de necessidades especiais possuem uma maior prioridade no atendimento. Foi feito um mapeamento desses atributos reais em atributos visuais da visualização. Mais relevante que os atributos reais escolhidos, é importante destacar que a visualização tem a capacidade de mostrar, de forma efetiva, até três atributos reais de uma mesma pessoa. Uma vez que o sistema de gerenciamento consiga coletar tal informação (ou outras) a visualização pode ser configurada para mudar o mapeamento.

A Tabela 2.1 descreve como foi feito o mapeamento real para o visual na VisEM. Pessoas representadas pela cor preta significam que estão imóveis ou que estão há muito tempo paradas no mesmo lugar. De forma arbitrária, foi definido que se as três últimas posições de uma pessoa - em três instantes de tempo sucessivos - forem iguais, a VisEM a representará como imóvel. Isto pode ser configurado no ambiente. A cor vermelha representa pessoas que estão em movimento ou que estão recentemente em uma posição. Para representar pessoas sem necessidades especiais, utilizou-se círculos. Triângulos foram utilizados para representar pessoas com necessidades especiais. $\mathrm{O}$ atributo visual tamanho (grande, médio e pequeno) foi mapeado para a faixa etária.

encontrados no site [3].

${ }^{2}$ Não faz parte do escopo deste trabalho definir como os dados serão capturados do ambiente. 


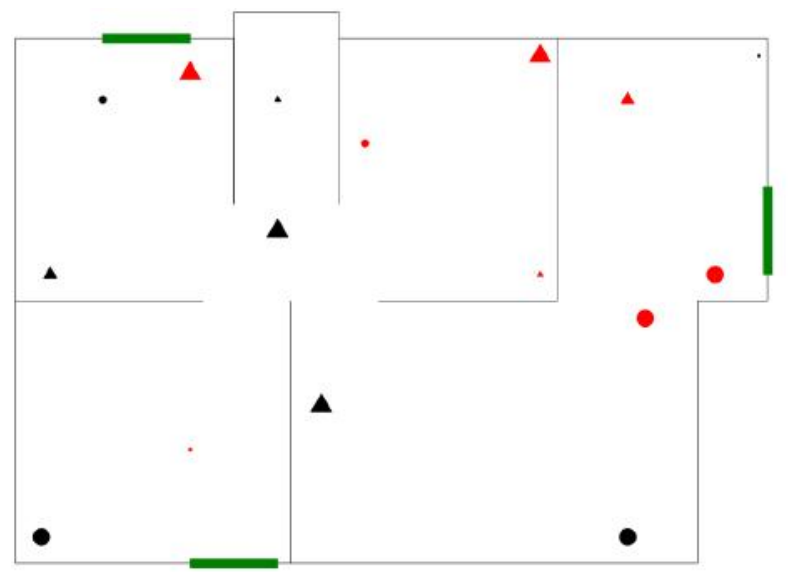

Figura 3: Representação visual de um ambiente indoor monitorado pela VisEM

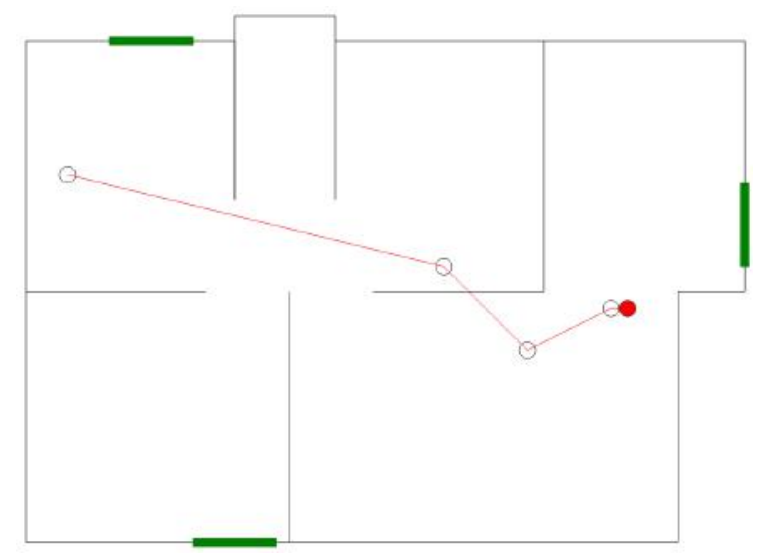

Figura 4: Histórico de posições de uma criança ou adolescente sem deficiência.

sistema utilizado pela VisEM possui dois eixos (X e Y), onde a posição das pessoas é projetada.

A VisEM utiliza círculos, triângulos, linhas e retângulos como elementos gráficos na elaboração das suas representações visuais. Das cinco propriedades gráficas descritas por Mazza [16], a VisEM utiliza as propriedades tamanho, cor e forma para representar pessoas de um ambiente fechado (conforme mapeamento apresentado na Tabela 2.1).

A Figura 3 é uma representação visual da planta baixa de um ambiente fechado, produzida pela VisEM. As retas pretas representam as paredes internas e externas do ambiente monitorado. Os retângulos de cor verde representam saídas de emergência. Para representar pessoas imersas no ambiente monitorado, a VisEM utiliza os atributos visuais já apresentados.

\subsubsection{Histórico de Posição das Pessoas}

A VisEM disponibiliza históricos de posições das pessoas em um ambiente indoor. A Figura 4 ilustra uma representação visual do histórico de uma criança ou de um adolescente sem deficiência. Os círculos sem cor representam as posições nas quais a criança esteve no ambiente indoor. O círculo vermelho é a posição atual da pessoa. Este círculo indica que a pessoa sem deficiência está há pouco tempo naquela posição.

As forças operacionais de resgate poderão perceber tendências espaciais através da representação visual do histórico das posições das pessoas. Por exemplo, suponha que haviam cincos pessoas - A, B, C, D e E - próximas uma das outras antes de uma emergência ocorrer em um ambiente fechado. Após um incidente, as pessoas B, C, D e E realizaram trajetos similares em direção a uma saída de emergência, enquanto a pessoa A permaneceu imóvel. Para salvar a pessoa A, a força operacional - por meio do histórico de posições das pessoas B, C, D e E - poderá inferir que existe uma grande possibilidade da pessoa A realizar um percurso similar ao das pessoas que eram vizinhas à pessoa $\mathrm{A}$ antes do incidente ocorrer.

\section{ESTUDO DE AVALIAÇÃO}

Esta seção apresenta em detalhes o estudo conduzido para avaliar a efetividade das visualizações produzidas pela VisEM. Todo o material experimental está disponível no website do estudo [3].

\subsection{Planejamento}

Objetivo. O principal objetivo do estudo foi avaliar a eficácia (O1) e eficiência (O2) dos recursos visuais utilizados na solução desenvolvida. Objetivos secundários foram definidos como sendo analisar, compreender e propor melhorias nas representações visuais para uma maior aplicabilidade ao contexto emergencial.

Participantes. O estudo foi realizado com dez participantes selecionados aleatoriamente. Um dos participantes era estudante da área de saúde, enquanto os demais eram estudantes de análise e desenvolvimento de sistemas. O participante mais novo tinha 20 anos de idade e o mais velho 33 anos. Dos dez participantes, seis eram do sexo masculino e quatro do sexo feminino.

Questionário. Os participantes responderam a um questionário contendo questões objetivas e subjetivas, detalhadas a seguir.

Questões Objetivas. As questões objetivas foram divididas em três grupos: Momento 1 (M1), Momento 2 (M2) e Momento 3 (M3). Cada Momento era composto de uma representação visual da VisEM, contendo dados fictícios de uma situação emergencial em um ambiente fechado. Cada Momento continha cinco itens descrevendo grupos de pessoas. A seguinte lista com itens de a) a e) correspondem ao Momento 1 do estudo.

a) criança/adolescente, sem deficiência, muito tempo nesta posição.

b) adulto, sem deficiência, pouco tempo nesta posição.

c) idoso, deficiente, muito tempo nesta posição.

d) criança/adolescente, sem deficiência, pouco tempo nesta posição.

e) idoso, sem deficiência, muito tempo nesta posição.

O participante deveria relacionar as alternativas às pessoas representadas na planta baixa produzida pela VisEM. 
Em cada planta baixa, existiam vinte figuras geométricas aleatórias, que representam vinte pessoas imersas em um ambiente monitorado. Para cada alternativa dos Momentos, existia pelo menos uma pessoa representada na planta com as características descritas. O participante deveria marcar com a letra correspondente, na planta baixa apresentada, o local onde se encontrava cada um desse grupo de pessoas. Uma legenda foi fornecida para auxiliar as pessoas a responderem as questões objetivas, caso necessário.

Para calcular o desempenho dos participantes em cada item, utilizou-se uma fórmula que define a nota mínima atribuída ao participante para cada acerto ou erro: nota mínima $=1$ / quantidade máxima possível de itens certos de uma alternativa. Por exemplo, a alternativa b) tinha como resposta duas pessoas na planta baixa que possuíam tais características. A nota mínima, da alternativa b) é igual a 1 dividido por 2. Neste caso, para cada pessoa relacionada de forma correta na alternativa b), o participante do estudo ganharia $+0,5$. De forma análoga, para cada pessoa relacionada de forma incorreta, o participante ganharia -0,5. Desta forma, o desempenho máximo que um participante pode alcançar na alternativa b) foi +1 ponto. A pontuação final do Momento era a média aritmética dos cinco itens e a média final do participante era a média aritmética dos três momentos.

Para medir a eficiência, foi controlado o tempo de início e fim da realização de cada Momento para cada participante.

Questões Subjetivas. Nas questões subjetivas, buscou-se coletar opiniões e demais impressões dos participantes acerca da visualização. Para essas questões, foi feito uma entrevista gravada em áudio. A seguir, estão as perguntas feitas aos participantes:

1. Você acha que é fácil o entendimento da representação visual?

2. Você acha que é efetivo a representação visual para um contexto de salvamento em ambientes fechados?

3. Você tem alguma sugestão referente à representação visual?

Estudo Piloto. Para avaliar os instrumentos utilizados, foi conduzido um estudo piloto com duas pessoas. A partir disso, foi possível fazer alguns ajustes no material experimental.

\subsection{Execução}

O estudo foi executado nos dias 15 e 16 de outubro de 2015, nas salas do (removido devido ao blind review). Antes de responderem aos questionários, os participantes receberam explicações relacionadas aos objetivos do desenvolvimento da VisEM. Foi-lhes dito que os mesmos avaliariam representações visuais, as quais são fruto de um trabalho de pesquisa que tem como objetivo a criação de uma solução computacional para situações emergenciais. No treinamento, foi utilizado a representação visual do Momento 3. Os participantes compreenderam que o objetivo da representação visual é descrever a posição e as características de pessoas imersas em um ambiente monitorado.

Em seguida, ensinou-se aos participantes a maneira correta de responder as questões objetivas. Antes dos participantes responderem as questões, foi-lhes pedido que sinalizassem, com a letra da alternativa, as pessoas da planta que possuíssem as características da alternativa. Também

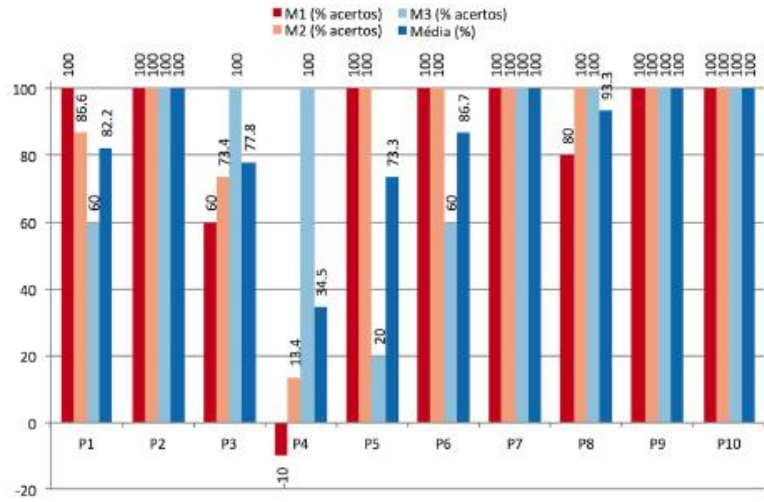

Figura 5: Percentual de acerto dos participantes.

foi-lhes dito que poderiam encontrar na planta uma ou mais pessoas que atendessem às características de uma alternativa. Os participantes foram instruídos a usarem a legenda para consulta apenas em último caso.

Cada participante respondeu o questionário individualmente, sob a supervisão dos experimentadores. Para cada Momento, eram fornecidos a representação visual e as alternativas. Após realizar as atividades de cada Momento, os participantes informavam se utilizaram ou não a legenda. $\mathrm{O}$ tempo gasto para responder cada Momento foi computado no estudo. Após as questões objetivas, a entrevista qualitativa foi conduzida. Por fim, foi aplicado um questionário de caracterização dos participantes.

\subsection{Resultados}

Eficácia (O1). A Figura 5 apresenta os acertos totais dos participantes (em média) para todos os momentos (M1, M2, M3), além da média global. No geral, o número de acertos foi bastante alto, próximo de $100 \%$ para a maior parte dos casos. Isto mostra que, apesar de ser o primeiro contato do participante com a representação visual, ele foi capaz de entendê-la facilmente e executar as atividades que lhes foram requeridas. Os participantes P2, P7, P9 e P10 tiveram um desempenho de $100 \%$. O participante P4 teve um desempenho diferente dos demais, chegando a ter um média negativa no Momento M1. Para os demais participantes, a média foi sempre superior a $60 \%$. A média global de desempenho entre todos os participantes (incluindo o outlier $\mathrm{P} 4$ ) em todos o momentos foi de $84,78 \%$.

Em relação ao uso da legenda, dos 10 participantes somente 3 fizeram uso desta. A partir da média de desempenho por participantes e o baixo índice de uso da legenda, pode-se inferir que as representações visuais, produzidas pela VisEM, são eficazes à percepção visual humana.

Eficiência (O2). A Figura 6 apresenta o tempo total dos participantes (média em minutos) para todos os momentos (M1, M2, M3), e a média global. No geral, o tempo gasto pelos participantes foi baixo. Os participantes gastaram, no pior caso, menos de 5.6 minutos para responder todas as alternativas de um determinado momento. Isto aconteceu com P1, P2 e P10 em M1, e P7 em M3. O participante P9 destacou-se como o mais rápido, na média, entre os respondentes, com 1.3 minuto de média global. Este mesmo parti- 


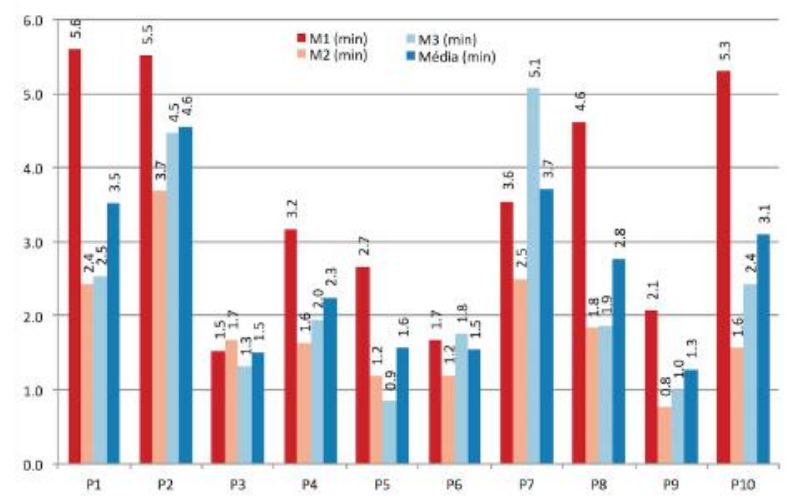

Figura 6: Tempo gasto pelos participantes.

cipante obteve um desempenho de $100 \%$. Tal fato permite inferir que as representações visuais da VisEM poderão ser utilizadas em contextos no qual se deseja uma informação rápida e precisa, como é o caso de situações de emergência. O participante P2 teve o maior tempo gasto entre os participantes, com 4.6 minutos em média.

Observa-se ainda que a maioria dos participantes gastou mais tempo no Momento 1, foi mais rápido no Momento 2 e aumentou seu tempo total no Momento 3. O tempo médio gasto no Momento 1 foi maior que no Momento 3. Esperavase que o tempo fosse decrescendo do momento M1 para o M3, uma vez que o participante estivesse mais confortável com o uso da visualização. Entretanto, o Momento 2 teve, no geral, o menor tempo entre os participantes. Isto pode ter acontecido pelo fato deste cenário ser menos complexo ao processo cognitivo humano que os demais.

Avaliação qualitativa. A Tabela 2 apresenta as respostas dos participantes para as duas primeiras perguntas subjetivas. Dos 10 participantes, 7 disseram que a representação visual da VisEM é de fácil entendimento. 9 participantes consideraram efetiva ao contexto de salvamento. Por outro lado, metade dos entrevistados disseram que o tamanho das figuras geométricas é ruim quando precisavam identificar a faixa etária. A solução sugerida por eles foi alterar o tamanho das figuras, ou aumentar a discrepância entre os tamanhos. Um dos participantes sugeriu o uso de símbolos diferentes para cada faixa etária.

\subsection{Ameaças à validade}

Buscou-se neste estudo avaliar a efetividade da VisEM do ponto de vista de sua eficácia e eficiência no contexto de um gerenciamento de emergência em ambiente fechado. Um risco à validade diz respeito a característica do estudo. Não existe, neste caso, um conjunto de dados real que pudesse ser usado como base para comparar a eficácia e eficiência dos resultados. De fato, tais aplicações são difíceis de se validar in vivo (condições realistas). Para tal, seria necessário simular de fato alguma emergência em ambientes fechados e verificar se o centro de comando conseguiria, de forma mais eficaz e mais eficiente, atuar no salvamento. Mesmo assim, acredita-se que a VisEM pode ser usada de forma efetiva para transmitir o contexto de uma emergência indoor, dado os altos índices de acertos em curtos espaços de tempo.

\begin{tabular}{lll}
\hline Participante & Q1 & Q2 \\
\hline \hline P01 & Sim & Sim \\
\hline P02 & Não & Não \\
\hline P03 & Sim & Com certeza \\
\hline P04 & Não & Sim \\
\hline P05 & Sim & Com certeza \\
\hline P06 & Não & Sim \\
\hline P07 & Sim & Sim \\
\hline P08 & Sim & Sim \\
\hline P09 & Sim & Sim \\
\hline P10 & Sim & Sim \\
\hline
\end{tabular}

Tabela 2: Respostas das $1^{\mathrm{a}}$ e $2^{\mathrm{a}}$ questões subjetivas.

\section{NOVA REPRESENTAÇÃO VISUAL}

O estudo conduzido destacou possibilidades de melhoria para a VisEM. Alguns participantes reportaram que a visualização poderia ser modificada. Eles tiveram dificuldades principalmente em diferenciar o atributo visual tamanho, mapeado para a faixa etária das pessoas presentes no ambiente. Eles disseram que o tamanho do idoso e do adulto são confundíveis e leva-se tempo para aprender. Os participantes disseram que necessitaram de uma referência para identificar as faixa etárias. Apesar de conseguirem identificar os três tamanhos, foi reportado que esta era a tarefa de maior esforço cognitivo.

Além disso, não há uma ordem linear natural na prioridade de resgate por faixa etária. Nos estudos preliminares, acreditava-se que a ordem de prioridade seria criança/adolescente, adulto, idoso. Desta forma, o atributo tamanho - eficiente para dados ordinais - com valores pequeno, médio e grande poderiam ser linearmente associados as faixas etárias. Com o avanço dos estudos junto aos especialistas, percebeu-se que a prioridade era diferente: criança/adolescente, idoso, adulto (ver Seção 2.1), perdendo assim a ordem linear natural. Ou seja, de acordo com as técnicas de gerenciamento de emergência, a ordem de salvamento difere da ordem do crescimento biológico humano. Consequentemente, era natural de se esperar que o atributo tamanho não seria o mais adequado para representar dados categóricos [16]. Como o estudo já tinha sido planejado, optou-se por não ajustar o seu planejamento.

Após a execução do estudo, foi feito uma nova proposta de visualização. O novo mapeamento de dados em atributos visuais pode ser visto na Tabela 3 . O atributo visual cor continuou mapeado da mesma forma: preto para representar uma pessoa que está parada há um determinado tempo no mesmo local, e vermelho, para o caso oposto. $\mathrm{O}$ atributo forma ganhou mais um valor (quadrado) e passou a representar a faixa etária. Agora, cada faixa etária é representada por uma forma diferente: triângulo representando crianças e adolescentes, círculo representando adultos e quadrado representando idosos. Por fim, o atributo visual marcador adicional foi utilizado para representar uma pessoa com necessidade especial. Esse atributo, adequado para representar dados categóricos [16], é representado pela adição de uma marca em um conjunto de objetos para ressaltar um 


\begin{tabular}{lll}
\hline $\begin{array}{l}\text { Atributo } \\
\text { Visual }\end{array}$ & Descrição Visual & Atributo Real \\
\hline \hline Cor & Preta / Vermelha & $\begin{array}{l}\text { Parado / Em } \\
\text { movimento }\end{array}$ \\
\hline Forma & Triângulo / & Criança / Idoso / \\
& Quadrado / Círculo & Adulto \\
\hline $\begin{array}{l}\text { Marcador } \\
\text { adicional }\end{array}$ & Traço preto & Com necessidade \\
& & especial \\
\hline
\end{tabular}

Tabela 3: Novo mapeamento dos dados de pessoas a atributos visuais.

objeto em particular.

A Figura 7 apresenta um exemplo da nova solução visual da VisEM para representar pessoas imersas em ambiente $i n$ door. Apesar de ter sido construída levando em consideração o feedback dos participantes do estudo apresentado na Seção 3, não pode-se fazer inferência a respeito de sua efetividade, uma vez que esta nova representação visual não foi avaliada até então.

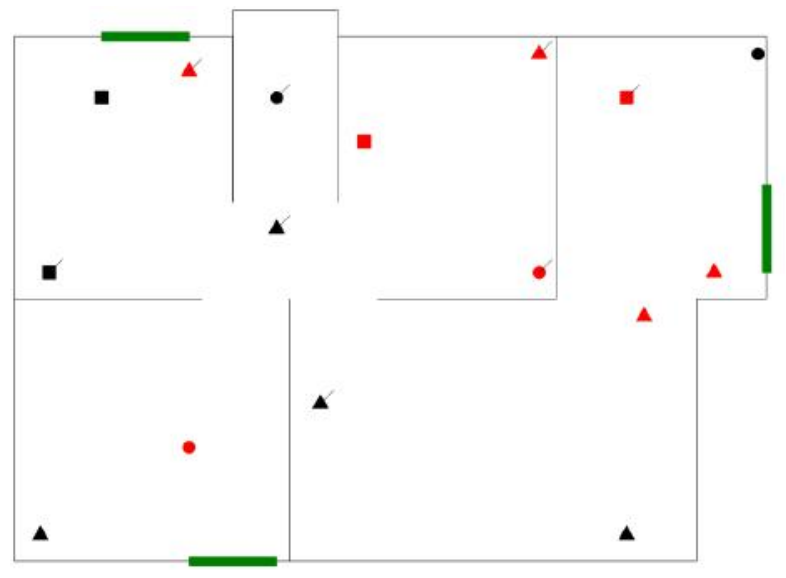

Figura 7: Nova representação visual da VisEM.

\section{TRABALHOS RELACIONADOS}

Trabalhos sobre gerenciamento de emergências buscam tanto a identificação de pessoas no ambiente sendo monitorado quanto a representação destas. Destaca-se aqui, nesta seção, as representações visuais utilizadas em alguns trabalhos encontrados na literatura.

Lopes [14] desenvolveu um projeto de um ambiente 3D de visualização e reprodução de eventos capturados e interpretados a partir de ambientes físicos cientes de contexto para aplicações de preparação para emergências. A partir deste ambiente é possível obter-se o histórico de posição de pessoas, a localização de pessoas e a localização de saídas de emergência, mas a visualização poderá se tornar complexa e imprecisa, já que pessoas ou objetos poderão estar ocultos atrás de outros objetos ou pessoas. Segundo Mazza [16], "alguns estudos empíricos têm mostrado que representações 3D aumentam a carga cognitiva, ou o esforço mental do usuário para corretamente interpretar os dados representados". No sistema descrito por Lopes [14], uma imagem bem mapeada gera uma representação visual muito parecida com as coisas do mundo real. Se a representação visual estiver sobrecarregada de informações, as relações entre os elementos mapeados serão mais difíceis de ser compreendidas. Consequentemente, a representação terá um baixo nível de aprimoramento perceptual.

Oliveira [20] descreve em seu trabalho um sistema de criação e visualização de interfaces do usuário cientes de contexto para realidade aumentada. Usa-se nesse trabalho tanto 2D quanto 3D. Entretanto, a combinação de representações visuais $2 \mathrm{D}$ e $3 \mathrm{D}$ pode tornar a informação mais complexa, sobrecarregando a cognição de quem a vê.

Em [19], os autores propõem um framework web para visualizações indoor/outdoor de sistemas de sensoriamento. A visualização indoor é feita através de uma planta cadastrada previamente com o objetivo de mostrar onde estão localizados os sensores dentro da construção. Em [13], foi desenvolvida uma visualização através de um mapa 3D para apresentar dados de rastreamento de localização em ambientes indoor. O sistema armazena a localização do usuário e invoca o mapa 3D baseado na posição estimada do usuário, mostrando e atualizando a sua posição no ambiente.

Em [5], os autores propõem o uso de uma visualização 3D para rastreamento de localização em ambientes indoor. O principal objetivo desse trabalho é complementar a capacidade de rastreamento e localização das redes de sensores sem fio fornecendo localizações 3D precisas. Em [10], foi desenvolvida uma abordagem sobre os sistemas de localização indoor no contexto de emergência. Essa abordagem baseouse na tecnologia RFID para coletar os dados do ambiente. Embora tenha sido proposto um sistema para localização em ambientes indoor, o trabalho não implementa nenhuma representação visual do ambiente. Em [8], os autores apresentam um sistema para mapeamento de ambientes indoor baseado em câmeras. A ferramenta realiza o mapeamento do ambiente rastreando pontos de referência nas imagens captadas pela câmera. As visualizações foram geradas pelas câmeras de visão estéreo que permitiram representar as paredes do ambiente indoor mapeado.

Os trabalhos acima apresentaram algumas das abordagens sobre ambientes indoor. Entretanto, pode-se perceber que o principal objetivo desses trabalhos é a aplicação de técnicas de identificação via sensores, localização e rastreamento em ambientes fechados. Alguns deles apresentam representações visuais para visualizar os dados coletados do ambiente indoor, porém, não foi encontrado nenhum trabalho que fizesse avaliação da efetividade da visualização proposta.

\section{CONCLUSÃO}

O ser humano passa muito tempo em locais fechados. É importante ter tecnologias que possam dar suporte às pessoas em caso de um emergência nestes ambientes. Dois desafios podem ser destacados: identificar as pessoas e visualizar o ambiente sendo monitorado. Observa-se que muitos trabalhos focam seus esforços no primeiro. Apesar da relevância (e do desafio) da identificação, é importante também investigar como prover visualizações do ambiente fechado de forma que a informação seja transmitida para uma força de resgate de forma eficaz e eficiente.

Este trabalho apresentou um estudo conduzido para conceber, construir e validar uma representação visual de um 
ambiente fechado no contexto de um gerenciamento de emergência. Foi produzida uma solução chamada VisEM, que teve como objetivos ser eficaz, eficiente, priorizar o resgate e ser flexível.

Para priorizar o resgate, foram conduzidos estudos com especialistas da área para entender como deve acontecer a priorização de resgate. A visualização foi projetada para ser configurável e mostrar diferentes atributos reais de pessoas através de seus atributos visuais. Desta forma, o operador poderá ter uma visão do cenário de emergência, priorizando seu antedimento caso necessário.

A VisEM faz parte de um sistema de gerenciamento de emergência (Rescuer Indoor - RI) que, além da visualização, realiza também a identificação de pessoas no ambiente. Apesar de ser parte do RI, a VisEM foi construída para ser flexível, de forma que pudesse ser utilizada em qualquer sistema de gerenciamento de emergências. Para avaliar sua efetividade do ponto de vista da eficácia e eficiência, foi conduzido um estudo de compreensão do ambiente fechado com um grupo de participantes. Os participantes tiveram um desempenho bastante satisfatório, tendo em alguns casos $100 \%$ de acurácia em suas respostas. Pouco tempo foi necessário para atingir os objetivos descritos nas tarefas do estudo. Desta forma, acredita-se que a VisEM é efetiva para o contexto de gerenciamento de emergência em ambientes fechados.

A partir do estudo, foi possível observar algumas limitações na solução visual desenvolvida. Isto ocorreu principalmente no que diz respeito ao mapeamento visual (atributo real para visual) implementado na solução. Uma nova solução visual para a VisEM já foi projetada, a qual será avaliada futuramente. Como trabalhos futuros, pretende-se realizar uma nova avaliação da VisEM e incrementar o ambiente com funcionalidades tais como a visualização de rotas de fuga, com o objetivo de ajudar as forças operacionais em suas atividades de salvamento em ambientes fechados.

\section{REFERÊNCIAS}

[1] About. http://paperjs.org/about/. Acessado em 29 de agosto de 2015

[2] Jquery. http://jquery.com. Acessado em 29 de agosto de 2015.

[3] Visem wiki page. https://rescue.ifba.edu.br/visem/index.html. Accessed: 2016-04-04.

[4] I. Al-Naimi, C. B. Wong, P. Moore, and X. Chen. Advanced approach for indoor identification and tracking using smart floor and pyroelectric infrared sensors. In Information and Communication Systems (ICICS), 2014 5th International Conference on, pages 1-6, April 2014.

[5] M. Bal, H. Xue, W. Shen, and H. Ghenniwa. A 3-d indoor location tracking and visualization system based on wireless sensor networks. In Systems Man and Cybernetics (SMC), 2010 IEEE International Conference on, pages 1584-1590. IEEE, 2010.

[6] C. Bueno. Sua casa é um ambiente propício para alergias? Faça o teste e descubra. UOL, São Paulo, 2013.

[7] F. Dusse, P. S. Júnior, A. T. Alves, R. Novais, V. Vieira, and M. Mendonça. Information visualization for emergency management: A systematic mapping study. Expert Systems with Applications, 45:424 - 437, 2016.

[8] E. Emilsson and J. Rydell. Chameleon on fire - thermal infrared indoor positioning. In 2014 IEEE/ION Position, Location and Navigation Symposium-PLANS 2014, 2014.

[9] D. Garlan, F. Bachmann, J. Ivers, J. Stafford, L. Bass, P. Clements, and P. Merson. Documenting Software Architectures: Views and Beyond. Addison-Wesley Professional, 2nd edition, 2010.

[10] R. Giuliano, F. Mazzenga, M. Petracca, and M. Vari. Indoor localization system for first responders in emergency scenario. In Wireless Communications and Mobile Computing Conference (IWCMC), 2013 9th International, pages 1821-1826. IEEE, 2013.

[11] J. Hightower and G. Borriello. Location systems for ubiquitous computing. Computer, 34(8):57-66, Aug. 2001.

[12] P. S. Júnior, R. Novais, V. Vieira, L. Pedraza, M. Mendonça, and K. Villela. Visualization mechanisms for crowdsourcing information in emergency coordination. In XIV Brazilian Symposium on Human Factors in Computer Systems, pages 1-10. TeX Users Group, March 2015.

[13] B.-G. Lee, Y.-S. Lee, and W.-Y. Chung. 3d map visualization for real time rssi indoor location tracking system on pda. In Convergence and Hybrid Information Technology, 2008. ICCIT'08. Third International Conference on, volume 1, pages 375-381. IEEE, 2008

[14] A. R. Lopes. Projeto de um Ambiente 3D de Visualização e Repredução de Eventos Capturados e Interpretados a Partir de Ambientes Físicos Cientes de Contexto para Aplicações de Preparação para Emergência. UFSCar, São Carlos, 2006.

[15] C. A. P. Lugon. Curso de formação de bombeiro profissional civil - gerenciamento de emergências. Centro de Ensino e Instrução de Bombeiros - Seção de Cursos de Extensão, Serra-ES.

[16] R. Mazza. Introduction to Information Visualization. Springer, Lugano, 2009.

[17] L. Narciso, A. Maslinkiewicz, and D. R. J. de Freitas. Levantamento de Doenças Respiratórias e sua Associação com Ambientes Climatizados na Comunidade da Universidade do Oeste de Santa Catarina (UNOESC) de Xanxerê. Unoesc \& Ciência ACBS, 2014.

[18] N. Pirzada, M. Y. Nayan, F. S. M. F. Hassan, and M. A. Khan. Device-free localization technique for indoor detection and tracking of human body: A survey. Procedia - Social and Behavioral Sciences, 129:422 - 429, 2014. 2nd International Conference on Innovation, Management and Technology Research.

[19] M. Simek, L. Mraz, and K. Oguchi. Sensmap: Web framework for complex visualization of indoor \& outdoor sensing systems. In Indoor Positioning and Indoor Navigation (IPIN), 2013 International Conference on, pages 1-5. IEEE, 2013.

[20] Állan César Moreira de Oliveira. Criação e Visualização de Interfaces do Usuário Cientes de Contexto para Realidade Aumentada. UFSCar, São Carlos, 2011. 